\title{
Inheritance of duplicated fumarase and phosphoglucomutase loci in lake trout (Salvelinus namaycush)
}

\author{
J. Ellen Marsden*, \\ Charles C. Krueger*, \\ Harold L. Kincaid $\dagger$ and \\ Bernie May
}

\author{
* Department of Natural Resources, College of \\ Agriculture and Life Sciences, Fernow Hall, Cornell \\ University , Ithaca, NY 14853, U.S.A. \\ $\dagger$ National Fishery Research and Development \\ Laboratory, U.S. Fish and Wildlife Service, R.D. \# 4, \\ Box 47, Wellsboro, PA 16901, U.S.A. \\ $\ddagger$ Cornell Laboratory for Ecological and Evolutionary \\ Genetics, Section of Ecology and Systematics, \\ Langmuir Laboratory, Cornell University, Ithaca, NY \\ 14853, U.S.A.
}

Two polymorphic, duplicated loci (fumarase-1,2 and phosphoglucomutase-3,4), previously undescribed in salmonids, were examined in lake trout to determine their electrophoretic expression, mode of inheritance, and linkage associations. Both loci appear to be located on chromosomes which show residual tetrasomy. Neither locus pair shows joint segregation with other loci examined or with each other.

\section{INTRODUCTION}

Electrophoretic data are widely used for obtaining genetic information about fish populations. Such information has been used in stock identification, genetic tagging, measures of inbreeding in hatchery broodstocks, and the detection of triploids (e.g., Allendorf and Utter, 1979; Grant et al., 1980; Shaklee, 1983; Purdom et al., 1976).

Electrophoretic analyses of lake trout ( $\mathrm{Sal}$ velinus namaycush) populations present three problems: (a) this species has lower genetic variability at isozyme loci relative to other salmonid species, (Allendorf and Utter, 1979; Dehring et al., 1981), (b) the electrophoretic banding patterns are complicated due to the tetraploid ancestry of the salmonids (May, 1980), which is reflected in an increased number of loci coding for metabolic enzymes relative to related diploid Salmoniformes, and (c) residual associations between homeologous chromosomes can result in exchange of alleles between loci (i.e., isoloci) located distal to the centromere and in the apparent linkage of non-syntenic disomic loci (pseudolinkage; Davisson et al., 1973; Wright et al., 1983).

In order to identify additional genetic markers for population studies on lake trout, an extensive survey of isozyme loci was performed in our laboratory (Krueger et al., in prep.). This survey revealed polymorphisms for fumarase (FUM) and a new phosphoglucomutase (PGM) zone of activity which have not been described in salmonids. The present study describes the electrophoretic expression and mode of inheritance of these loci.

\section{METHODS}

Superior (Marquette) and Seneca Lake strains of lake trout were obtained from the Tunison Nutrition Fish Laboratory (TNFL) in Cortland, NY, and from the Allegany National Fish Hatchery near Warren, PA. Crosses were made on 24 October and 1 November 1984 at the National Fishery Research and Development Laboratory (NFRDL) near Wellsboro, PA, and on 12 November 1984 at TNFL. All crosses were produced by stripping and storing gametes from individual fish, which were then sacrificed and sampled for muscle, liver, kidney, heart, and eye tissue. These tissues were analysed electrophoretically within hours of tissue and gamete collection. Based on identification of individual genotypes, parental mating pairs were selected and gametes were combined within 12 hours of collection. All test lots were incubated and raised to the fingerling stage at NFRDL. Each test lot (family) was labelled using 
numbers to designate the female parent and letters to designate the male parent. Fry were sacrificed after they reached a minimum length of approximately $6.0 \mathrm{~cm}$. Eighty individuals were sampled from each family, except crosses 10-A $(N=40)$, 13-D $(N=72)$, and 99- $H H(N=170)$, and were frozen whole at $-80^{\circ} \mathrm{C}$. Prior to electrophoresis, fry were sampled for muscle, kidney and eye tissue. Electrophoretic procedures, staining recipes, interpretation of banding patterns, and nomenclature followed May et al. (1979b) and Allendorf et al. (1977). Alleles were designated by numbers, with the most common allele being ' 1 '. Arrangement of alleles at duplicated loci, when it was known, was designated in the format $11 / 22$, where the slash separates alleles from different homeologous pairs. Mobilities of allelic products at Aat-1,2, Mdh-3,4, and $\mathrm{Me}-1,2$ coincided with those found in Lake Superior lake trout by Dehring et al. (1981), and allelic products at Pep-pap-1,2 appeared to coincide with those found by Hollister et al. (1984). For ease of data comparisons between laboratories, we have used the mobilities given by these authors (table 1). Resolution of FUM and PGM was markedly improved by using an agar overlay in addition to the standard stain recipes; FUM could only be scored consistently using the agar overlay. The parental strains had previously been screened for over 90 loci (Krueger et al., in prep); therefore, potential parents were examined only at those loci which were variable in these strains. Fry were examined electrophoretically at those loci which were polymorphic in the parents and which provided information on single locus and/or joint segregation (table 1).

Data were analysed using a linkage analysis program modified from Suiter et al. (1983). Crosses were analysed for single locus segregation and joint segregation as described in May et al. (1979b), except as noted in text.

\section{RESULTS}

\section{Enzyme systems}

Salmonid isozyme banding patterns have been described in detail by May (1980) and Johnson (1984) for all polymorphic loci used in this study except for those coding for FUM and PGM, which are described below.

Fum-1,2: Two identical anodal zones of fumarase activity were found for these loci in muscle tissue (fig. 1). Since the two zones showed parallel patterns in all phenotypes observed, we presumed

Table 1 Enzyme systems used in this study, with the tissues and buffer systems on which they were best resolved

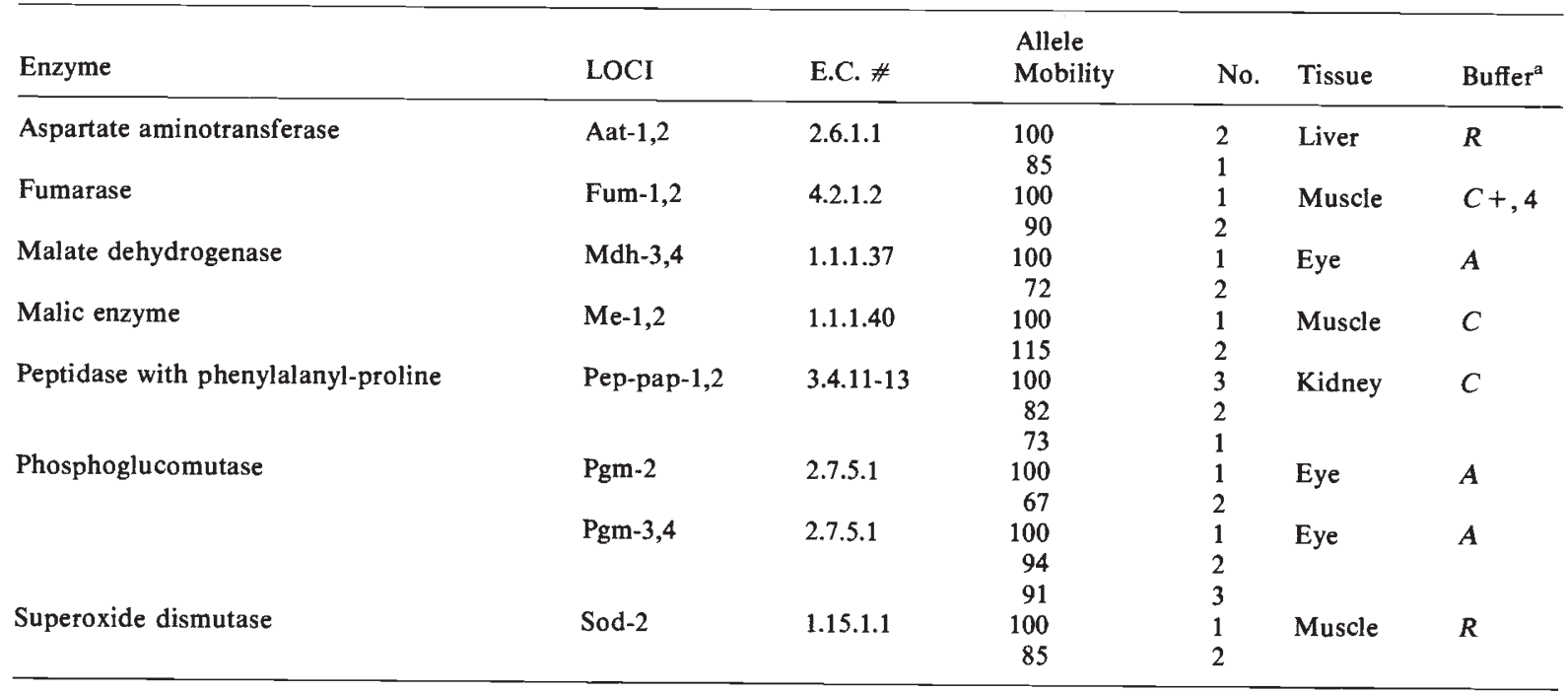

\footnotetext{
${ }^{\text {a }}$ Buffer systems used;

$R$, Ridgway et al., 1970

$C, C T$ of May et al., $1979 b$

$C+, C$ (above) with the $p \mathrm{H}$ raised to 7.0 with $\mathrm{NaOH}$

$A, A Z M$ of May et al., $1979 b$

4, continuous tris-citrate type $I$ of Selander et al., 1971
} 


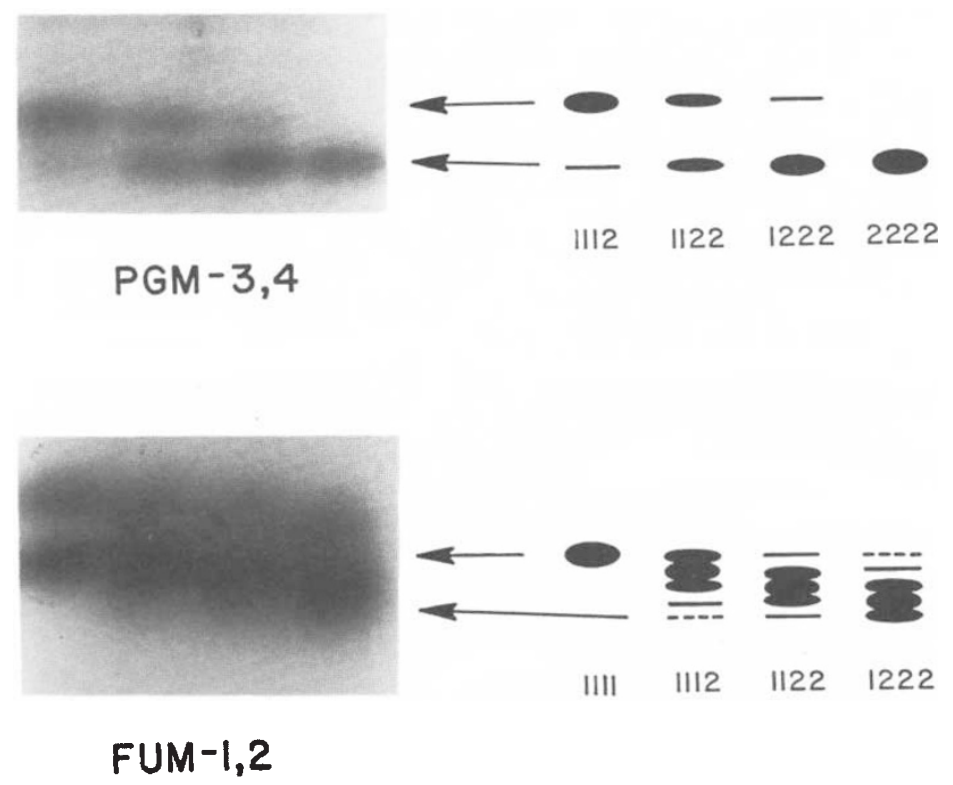

Figure 1 Photos and diagrams of electrophoretic banding patterns of fumarase-1,2 and phosphoglucomutase-3,4.

one of the two zones was an artifact; we consistently read the lower zone. Band density ratios conformed to those expected for a tetrameric enzyme encoded by four gene doses with two alleles.

$P g m-3,4$ : Five zones of activity were noted in eye tissue, one on the cathodal side of the origin and four on the anodal side. The cathodal zone corresponded to Pgm-1, and the slowest of the anodal zones was Pgm-2. The two central anodal zones are probably not PGM activity. The fastest of the anodal zones showed banding patterns which conformed to those expected for a monomeric enzyme coded by duplicate loci (see fig. 1). This zone was Pgm-3,4, described in muscle tissue by May et al. (1980). Three alleles were noted for Pgm-3,4, plus a null allele class. Null alleles $(\emptyset)$ were recognised on the basis of band intensity ratios; thus an individual with two copies of the 1 allele and one copy each of the 2 and $\emptyset$ allele would show bands at the 1 and 2 allelic product positions in a $2: 1$ ratio. An individual with more than one copy of a null allele would not be identifiable as having a null allele by visual examination of the phenotype, because band intensity ratios would conform to those expected for genotypes without null alleles. Null alleles have also been detected in salmonids at Mdh-1 (May et al., 1979a), Aat-1,2 (Stoneking et al., 1981a), Idh-4, Me-2, and Pgm-1 (Stoneking et al., 1981b). Stoneking et al. (1981a) discuss in detail the problems involved with interpreting gel banding patterns at a locus with a null allele.

\section{Single locus segregation}

In order to examine the inheritance mode of duplicated loci, it is necessary to determine that individual alleles segregate in a Mendelian fashion. All crosses which had a single copy of any allele at either Fum-1,2 or Pgm-3,4 were tested for nonrandom distribution of the allele in the progeny. In the absence of meiotic drive or differential survival of progeny, each allele should appear in 50 per cent of the progeny. Abberant single locus segregation was noted for Pgm-3,4 in one individual, the female in cross $10-A(p=0.011$; see table 2), but due to the number of simultaneous tests performed (23 crosses), this deviation was not considered significant (Cooper, 1968). Progeny from crosses with a single copy of any allele at Fum-1,2 or Pgm-3,4 were also examined for evidence of double reduction, which would result in an individual carrying two doses of the rare allele (Burnham, 1962). This was not noted for either of the duplicated loci.

Fum-1,2: only three crosses (9-B, 9-E, and 99 $H H$ ) had genotypes appropriate for determining whether inheritance of Fum-1,2 is other than disomic, i.e., a homozygote (1111 or 2222) or asymmetrical heterozygote (1112 or 1222) crossed with a symmetrical heterozygote (1122) (see table 2). The first two crosses used the same symmetrically heterozygous (1122) female (\#9), and in both crosses the progeny could have only been produced with all heterozygous (12) gametes coming from 
Table 2 Single locus segregation of fumarase and phosphoglucomutase in lake trout. Where it was possible to test for more than one allele or combination of alleles in a family, the alleles being tested are underlined; offspring categories which were grouped together for analysis are shown with single, double or no underlining. An asterisk indicates significance differences from expected ratios at or below the 0.05 level

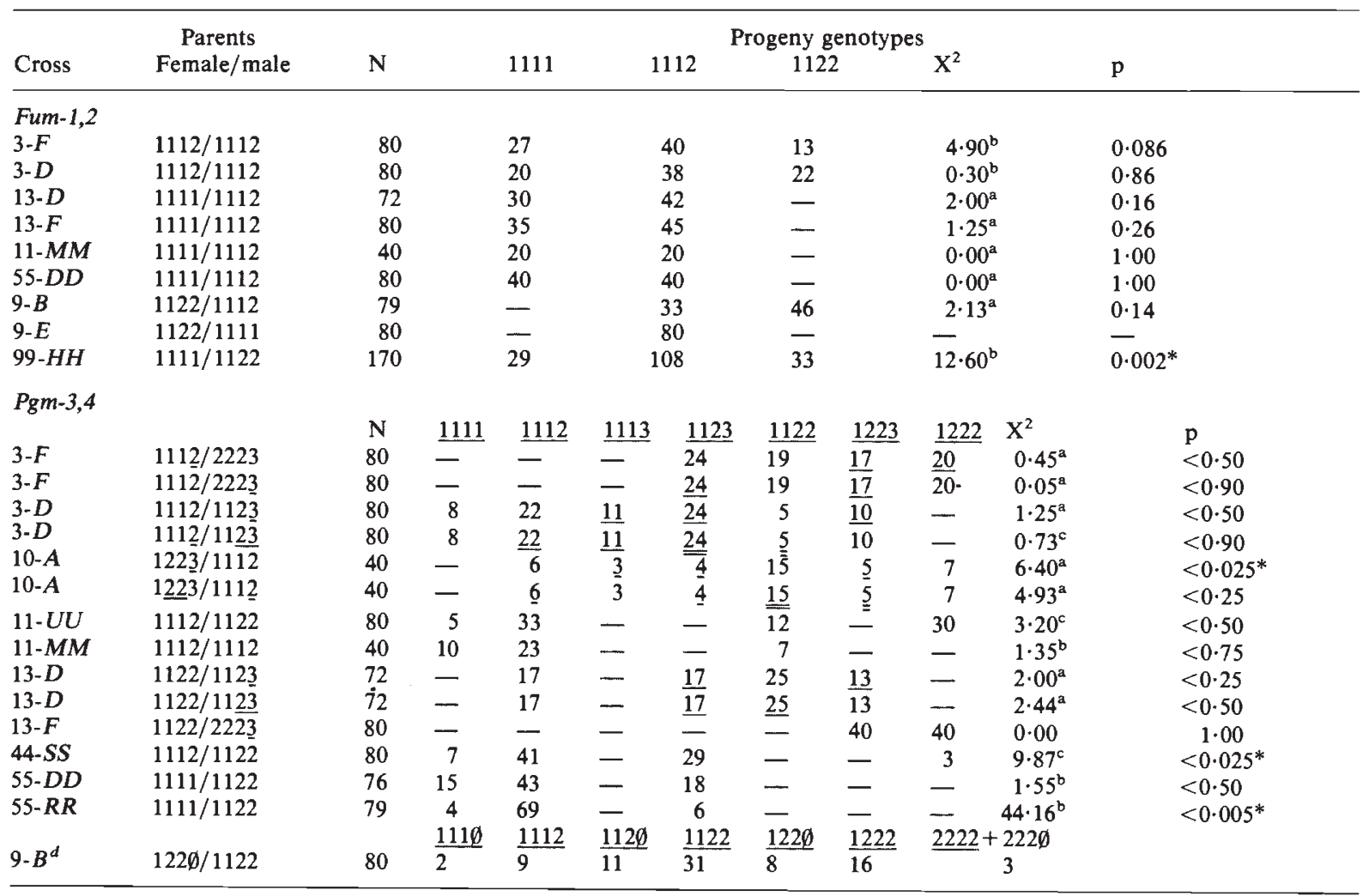

\footnotetext{
a tested against an expected $1: 1$ ratio with 1 d.f.

${ }^{b}$ tested against an expected $1: 2: 1$ ratio with 2 d.f.

${ }^{c}$ tested against an expected $1: 3: 3: 1$ ratio with 3 d.f.

d see text for explanation of this family.
}

the female parent. This implied that the female parent was homobivalent $(11 / 22-i . e$., the bivalent chromosome pairs were homozygous for different alleles, so that a 1 allele could only segregate with a 2 allele in meiosis). In cross $99-H H$ the male was symmetrically heterozygous and produced progeny that could only have been derived from three types of gametes: 11,12 , and 22 . Since he produced heterozygous gametes in excess of a $1: 2: 1$ ratio, he formed either random bivalents or heterobivalent tetravalents with crossing-over.

Pgm-3,4: Nine parents used in nine crosses had symmetrically heterozygous genotypes (see table 2 ). Of the three symmetrically heterozygous females, progeny phenotypes indicated that one was homobivalent, yielding only heterozygous gametes (in crosses 13- $D$ and 13-F) while the other two females were heterobivalent (crosses 10- $A$ and $9-B$ ). Three of the symmetrically heterozygous males produced an excess of heterozygous gametes, based on progeny phenotypes (crosses 9- $B, 44-S S$ and 55- $R R$ ). The remaining three males produced a $1: 2: 1$ ratio of $11: 12: 22$ gametes (crosses 3-D, 13-D, 55-DD, and 11- UU).

Cross 9- $B$ needs a detailed discussion, as it is the only cross which apparently included a null allele (table 2). The parental genotypes were $122 \emptyset$ for the female and 1122 for the male. If both parents were heterobivalent, 8 progeny classes would be produced $(1112,1122,1222,2222,111 \emptyset$, $112 \emptyset, 122 \emptyset$, and $222 \emptyset$ in a $1: 3: 3: 1: 1: 3: 3: 1$ ratio); however, only two $111 \emptyset$ or 1111 and four $222 \emptyset$ or 2222 progeny were produced, whereas the expected numbers were 5 and 10, respectively. (Note that $111 \emptyset$ and $222 \emptyset$ phenotypes are indistinguishable from homozygotes.) This suggests that the male was homobivalent $(11 / 22)$, and only produced 11 and 22 gametes through occasional 
tetravalent pairing and crossing-over. If this was the case, 1112 progeny should have been produced in small numbers and the remaining progeny classes $(112 \emptyset, 122 \emptyset, 1122$ and $122 \emptyset)$ would be expected in a $1: 1: 1: 1$ ratio. However, these genotypes are not reliably separable, as a $2: 1$ band ratio cannot always be readily distinguished from a $2: 2$ or $3: 1$ ratio. If the female was also homobivalent $(1 \emptyset ; 22)$ she could only have produced 12 and $2 \emptyset$ gametes. The presence of $111 \emptyset$ progeny and high numbers of 1222 and $112 \emptyset$ progeny indicate that she also produced $1 \emptyset$ and 22 gametes. Therefore she must have been heterobivalent $(12 / 2 \emptyset)$.

\section{Joint segregation}

All pairwise combinations of loci which yielded data on joint segregation are shown in table 3 (informative $N \geqq 40$ ). Detailed data will be provided upon request. Fumarase was tested for linkage with five other loci, including Pgm-3,4, in males and one other locus in females. Pgm-3,4 was also tested for linkage with four other loci, including Fum-1,2, in males, and two other loci in females. In one family $(13-D)$ it was possible to test for linkage between Pgm-3 and Pgm-4. There were no families in which it was possible to test for linkage between the two fumarase loci. Five tests showed significant deviation from independent segregation: Aat-1,2 with Me-1,2 in cross 3-F $(r=0.363)$; Pep-pap-1,2 with Me-1,2 in cross 9- $E(r=0.385)$; Fum-1,2 with Pgm-3,4 in cross $13-D(r=0.389)$; Me-1,2 with Pgm-3,4 in cross 3-F $(r=0.372)$; and Fum-1,2 with Pgm-3,4 in cross 3-F $(r=0 \cdot 325)$. However, only one of these $r$ values fits the criteria of $r \leqslant 0.35$ established by May et al. $(1979 b)$ for detection of non-random assortment, and each of these pairs of loci are shown to be unlinked in other crosses. Given the large chromosome arm number in this species ( $N F=100$; Davisson, 1969) compared with the low number of pairs available for testing, the probability of finding linkage is quite low.

\section{DISCUSSION}

In order to determine the mode of inheritance of salmonid loci for prediction of progeny genotype ratios, it is first necessary to understand the structure of the salmonid genome-that is, the types of chromosome pairing and segregation which occur. In female salmonids only bivalent pairing of chromosomes is thought to occur in meiosis (Wright et al., 1983; Allendorf and Thorgaard, 1984; however, see Rex, 1984). Evidence indicates that these are homologous bivalents. If random bivalents formed (homologous and homeologous), it is highly likely that tetravalents would occur, since they are seen to occur in males. A symmetrically heterozygous female at a given locus will either be homobivalent $(11 / 22)$, producing only 12 gametes, or heterobivalent $(12 / 12)$, producing a $1: 2: 1$ ratio of 11,12 , and 22 gametes respectively. In contrast, males exhibit some residual multivalent associations in which crossing-over is inhibited near the centromere, as evidenced by duplicated loci known to be involved in multivalents which are fixed for alternate alleles (e.g., Ldh-3,4; Wright et al., 1983). Where crossing-over is not inhibited, that is, for genes distal to the centromere, apparently exactly one cross-over

Table 3 Tests for joint segregation of loci in lake trout. The numbers refer to the number of crosses which were diagnostic for joint segregation for a given pair of loci. Examinations in males are above the diagonal and in females are below the diagonal

\begin{tabular}{|c|c|c|c|c|c|c|c|}
\hline & Aat-1,2 & Mdh-3,4 & $\begin{array}{l}\text { Pep- } \\
\text { pap-1,2 }\end{array}$ & Fum-1,2 & $\mathrm{Me}-1,2$ & Pgm-3,4 & Sod-2 \\
\hline Aat $-1,2$ & & & 1 & 2 & & $1^{a}$ & \\
\hline Mdh-3,4 & & & & 2 & & 3 & 2 \\
\hline Pep-pap-1,2 & & & & 2 & 1 & & \\
\hline Fum-1,2 & 2 & & & & $4^{b}$ & 2 & \\
\hline $\mathrm{Me}-1,2$ & 1 & & 1 & & & & \\
\hline Pgm-3,4 & 1 & & & & & & 2 \\
\hline Sod-2 & & & 1 & & & 1 & \\
\hline
\end{tabular}

a a single examination was made in which one of the Aat loci was tested against both of the Pgm loci in a single male (cross 13- $D$ ).

$b$ a single examination was made in which one of the Fum loci was tested against both of the Pgm loci in a single male (cross 13-D). 
occurs per chromosome arm pair (Allendorf et al., in prep., Thorgaard et al., 1983). In addition, of the three possible types of chromosome segregation in a tetravalent (Burnham, 1962), primarily alternate segregation occurs in salmonids. Double reduction progeny, which result from adjacent II pairing, are seen very rarely (Wright et al., 1983). If no crossing-over takes place, a homobivalent male who forms tetravalents will produce only heterozygous (12) gametes (see table 4). If single crossovers always occur between each chromosome pair, he will produce 11,12 , and 22 gametes in a $1: 2: 1$ ratio. The chromosomes in a heterobivalent individual can take either of two arrangements in a tetravalent, with the like alleles being either paired or unpaired (see fig. 2). If no cross-overs occur, the former arrangement will produce only 12 gametes and the latter will produce 11 and 22 gametes in equal ratios; since both arrangements are formed randomly and therefore with equal frequency, 11,12 , and 22 gametes will be formed in a total ratio of $1: 2: 1$. If cross-overs do occur, when like alleles are paired only 12 gametes will be produced; when unlike alleles are paired, 11, 12, and 22 gametes will be produced in a $1: 2: 1$ ratio. The expected total gamete ratio will be $1: 6: 1$. Thus a locus which is close to the centromere (no gene-centromere crossing-over) on
Table 4 Expected ratios of 11, 12 and 22 gametes from a symmetrically heterozygous individual given various types of chromosome configurations, pairing modes, and crossing-over frequencies, and only alternate segregation. $A=$ like alleles paired, $B=$ unlike alleles paired (see fig. 2 ). Adapted from Wright et al., 1983.

\begin{tabular}{|c|c|c|c|}
\hline & Type & chromosom & airing \\
\hline & bivalent & tetr & lent \\
\hline & & $\begin{array}{l}\text { no } \\
\text { x-over }\end{array}$ & $\begin{array}{l}\text { single } \\
\mathrm{x} \text {-over }\end{array}$ \\
\hline $\begin{array}{l}\text { homobivalent } \\
(11 / 22)\end{array}$ & $0: 1: 0$ & $0: 1: 0$ & $1: 2: 1$ \\
\hline heterobivalent & $1: 2: 1$ & $1: 2: 1$ & $1: 6: 1$ \\
\hline$(12 / 12)$ & & A) $0: 1: 0$ & $0: 1: 0$ \\
\hline & & B) $1: 0: 1$ & $1: 2: 1$ \\
\hline
\end{tabular}

a chromosome which pairs tetravalently is functionally equivalent to a locus on a chromosome which only pairs bivalently. A locus distal to the centromere will occasionally be identifiable in homobivalent individuals by the production of double reduction gametes (11 and 22) via chromatid non-disjunction (Burnham, 1962; Wright et al., 1983). Such a locus may also be identifiable in heterobivalent individuals, due to the production of an excess of heterozygous gametes; however, mixed ratios of bivalent and

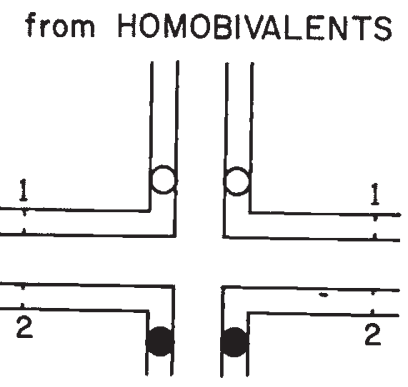

from HETEROBIVALENTS

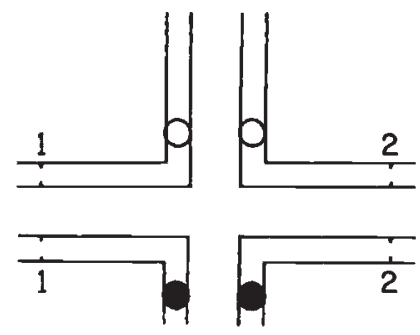

like alleles paired

A

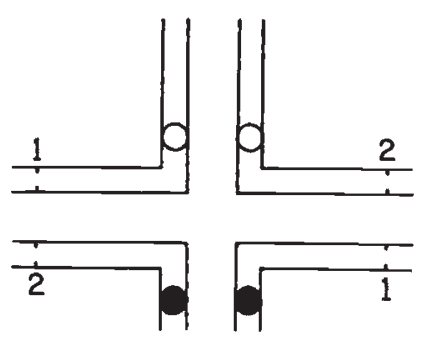

unlike alleles paired

B

Figure 2 Possible arrangements of alleles in a tetravalent in a symmetrically heterozygous individual. The two homologous chromosome pairs (denoted by solid or open-circle centromeres) which form the tetravalents can be homobivalent (both pairs homozygous for different alleles) or heterobivalent (both pairs heterozygous). 
tetravalent pairing in an individual forming homobivalents will also produce an excess of heterozygous gametes.

The results of this study indicated that the fumarase loci are probably located distal to the centromere on chromosomes which still show residual tetravalent pairing. The only female which was symmetrically heterozygous (used in crosses 9- $E$ and $9-B$ ) was homobivalent; the only symmetrically heterozygous male (cross $99-\mathrm{HH}$ ) produced an excess of heterozygous gametes, indicating either that he was heterobivalent with crossingover occurring between duplicate loci, or that he was homobivalent with mixed bivalent and tetravalent pairing.

Of the three females which were symmetrically heterozygous at Pgm-3,4 one was homobivalent and two were heterobivalent (segregating in a $1: 2: 1$ ratio). Of the six symmetrically heterozygous males, three produced 11,12 , and 22 gametes in a $1: 2: 1$ ratio, and three produced an excess of asymmetrically heterozygous gametes (table 3 ). These ratios indicate that $\mathrm{Pgm}-3,4$ must also be located on chromosome arms with tetravalent (homeologous) pairing of distally homologous regions. The variance in progeny ratios between families suggests that there were both homobivalent and heterobivalent males in the crosses, producing $1: 2: 1$ and $1: 6: 1$ gamete ratios, respectively. The low number of homozygous gametes produced by the male in cross $55-R R$ suggests that he was homobivalent; both tetravalent formation and crossing-over would be required to produce homozygous gametes from a homobivalent. Unpublished data from May and Wright indicates that gene-centromere distances for the fumarase and phosphoglucomutase systems are greater than 45 centimorgans in Salvelinus hybrids.

Additional information on these loci was provided by an electrophoretic analysis of 13 lake trout populations performed in our laboratory (in prep.). With respect to only the 1 and 2 alleles at Pgm-3,4, all five of the possible genotypes (1111, $1112,1122,1222$, and 2222) were seen in high numbers. This provides additional evidence that the two loci exchange alleles via homeologous pairing and crossing-over, since it is highly unlikely that two loci would carry the same alleles unless they had diverged recently. The low frequency of allele 2 at Fum-1,2 reduced the probability of finding individuals with more than two doses; however, a single population with a high frequency of this allele contained two 1222 individuals and one 2222 individual, which suggests that the loci do share alleles.
In summary, this study describes the inheritance of two previously undescribed polymorphic, duplicate loci-Fum-1,2 and Pgm-3,4. Data from these loci will be useful for future population and inheritance studies of lake trout and other salmonids. Data interpretation from inheritance studies must consider that exchange of alleles occurs within both locus pairs. The presence of a null allele class at Pgm-3,4 complicates the interpretation of gel banding patterns, as discussed by Stoneking et al. (1981).

Acknowledgements We thank Dave Ostergaard and Homer Zumstein at the Allegany National Fish Hatchery and Wayne Bonney and the staff of the Tunison Nutritional Fish Laboratory for their assistance in obtaining fish and making the crosses; the staff of the National Fishery Research and Development Laboratory for raising the fry; Christopher Azar and Kathrine Henley for technical support; and Steven P. Gloss of the New York Fish and Wildlife Cooperative Research Unit for support throughout the project. This work is a result of research sponsored by the NOAA Office of Sea Grant, U.S. Department of Commerce, under Grant \# NA85AADSG021. The U.S. Government is authorised to produce and distribute reprints for governmental purposes notwithstanding any copyright notation that may appear hereon. The work was also supported by the U.S. Fish and Wildlife Service through Research Work Order \#6 from the New York Fish and Wildlife Cooperative Research Unit, and by the National Fishery Research and Development Laboratory.

\section{REFERENCES}

ALLENDORF, F. W. AND THORGAARD, G. H. 1984 Tetraploidy and the evolution of salmonid fishes. Turner, B. J. (ed) In Evolution of the Salmonid Fishes, Plenum Press, New York, pp. 1-53.

ALlendorF, F. W. AND UTtER, F. M. 1979. Population genetics. (Hoar, W. S. and Randall, D. J. (eds.) In Fish Physiology, vol. 8, Academic Press, New York, pp. 407-454.

ALLENDORF, F. W., MITCHELL, N., RYMAN, N. AND STAHL, G. 1977. Isozyme loci in brown trout (Salmo trutta L.): detection and interpretation from population data. Hereditas, 86, 179-190.

ALLENDORF, F. W., SEEB, J. E., KNUDSEN, K. L., THORGAARD, G. H. AND LEARY, R. F. Gene-centromere mapping of 25 loci in rainbow trout. $J$. Heredity, in press.

BURnhaM, C. R. 1962. Discussions in Cytogenetics, Burgess Publishing Co., Minneapolis.

COOPER, D. W. 1968. The significance level in multiple tests made simultaneously. Heredity, 23, 614-617.

DAVISSON, M. T. 1969. Cytogenetic analysis of two teleost families, Salmonidae and Esocidae. Ph.D. thesis, Pennsylvania State University.

DAVISSON, M. T., WRIGHT, J. E. AND ATHERTON, L. M. 1973. Cytogenetic analysis of pseudolinkage of LDH loci in the teleost genus Salvelinus. Genetics, 73, 645-658.

DEHRING, T. R., BROWN, A. F., DAUGHERTY, C. H. AND PHELPS, S. R. 1981. Survey of the genetic variation among eastern Lake Superior lake trout (Salvelinus namaycush). Can. J. Fish. Aquat. Sci., 38, 1738-1747. 
GRANT, W. S., MILNER, G. B., KRASNOWSKI, P. AND UTTER, F. M. 1980. Use of biochemical genetic variants for identification of Sockeye salmon (Oncorhynchus nerka) stocks in Cook Inlet, Alaska. Can. J. Fish. Aquat. Sci., 37, 12361247.

HOLlister, A., JOHNSON, K. R. AND WRIGHT J. E. 1984. Linkage associations in hybridized Salvelinus genomes. $J$. Hered., 75, 253-259.

JOHNSON, K. R. 1984. Protein variation in Salmoninae: Genetic interpretations of electrophoretic banding patterns, linkage associations among loci, and evolutionary relationships among species. Ph.D. thesis, Pennsylvania State University.

MAY, B. 1980. The salmonid genome: evolutionary restructuring following a tetraploid event. Ph.D. thesis, Pennsylvania State University.

MAY, B., STONEKING, M. AND WRIGHT, J. E., JR 1979a. Joint segregation of malate dehydrogenase and diaphorase loci in brown trout (Salmo trutta). Trans. Am. Fish. Soc., 108, 373-377.

MAY, B, WRIGHT, J. E., JR AND STONEKING, M. 1979b. Joint segregation of biochemical loci in Salmonidae: results from experiments with Salvelinus and review of the literature on other species. J. Fish. Res. Board Can., 36, 1114-1128.

MAY, B, WRIGHT, J. E., JR AND STONEKING, M. 1980. Joint segregation of biochemical loci in Salmonidae. II. Linkage associations from a hybridized Salvelinus genome ( $S$. namaycush $\times S$. fontinalis). Genetics, 95, 707-726.

PURDOM, C. E., THOMPSON, D. AND DANDO, P. R. 1976. Genetic analysis of enzyme polymorphisms in plaice (Pleuronectes platessa). Heredity, 37, 193-206.

REX, J. D. 1984. Cytogenetic analysis of sparctic trout (Salvelinus alpinus $\times S$. fontinalis). MS thesis, Penn. State.

RIDGWAY, G. J., SHERBURNE, S. W. AND LEWIS, R. D. 1970. Polymorphism in the esterases of Atlantic herring. Trans. Am. Fish. Soc., 99, 147-151.
SELANDER, R. K., SMITH, M. H., YANG, S. Y., JOHNSON, W. E., AND GENTRY, J. B. 1971. Biochemical polymorphism and systematics in the genus Peromyscus. I. Variation in the Old-field mouse (Peromyscus polionotus). Studies in Genetics VI. Univ. Texas Publ. 7103.

SHAKLEE, J. B. 1983. The utilization of isozymes as gene marker in fisheries management and conservation. Rattazzi, M. C., Scandalios, J. G. and Whitt, G. S. (eds.) In Isozymes: Current Topics in Biological and Medical Research, vol. 11, Alan R. Liss, New York, pp. 213-247.

STONEKING, M., MAY, B. AND WRIGHT, J. E., JR $1981 a$ Loss of duplicate gene expression in Salmonids: Evidence for a null allele polymorphism at the duplicate aspartate aminotransferase loci in brook trout (Salvelinus fontinalis). Biochem. Genetics, 19, 1063-1077.

STONEKING, M., WAGNER, D. J. AND HILDEBRAND, A. C. $1981 b$. Genetic evidence suggesting subspecific differences between northern and southern populations of brook trout (Salvelinus fontinalis). Copeia, 1981, 810-819.

SUITER, K. A., WENDEL, J. F. AND CASE, J. S. 1983. Linkage-1. University of North Carolina, Chapel Hill, North Carolina.

THORGAARD, G. H., ALLENDORF, F. W. AND KNUDSEN, K. L 1983. Gene-centromere mapping in rainbow trout: high interference over long map distances. Genetics, 103, 771783.

WRIGHT, J. E., JR, JOHNSON, K., HOLLISTER, A. AND MAY, B 1983. Meiotic models to explain classical linkage, pseudolinkage and chromosome pairing in tetraploid derivative salmonids. Rattazzi, M. C., Scandalios, J. G. and Whitt, G. S. (eds.) In Isozymes: Current Topics in Biological and Medical Research, vol. 10, Alan R. Liss, New York, pp. 239-260. 